Escuela de Medicina, Universidad Peruana de Ciencias Aplicadas, Lima, Perú. Instituto Nacional de Salud, Lima, Perú. Escuela de Medicina, Universidad del Mar, Iquique, Chile.

${ }^{4}$ Sociedad Científica de Estudiantes de Medicina UPC (SOCIEMUPC), Lima, Perú. EEscuela de Medicina Luis Razetti, Facultad de Medicina, Universidad Central de Venezuela, Caracas, Venezuela. ${ }^{6}$ Departamento de Medicina Comunitaria, Facultad de Ciencias de la Salud, Universidad Tecnológica de Pereira, Pereira, Risaralda, Colombia. Oficina de Investigación Científica,

Cooperativa de Entidades de Salud de Risaralda (COODESURIS), Pereira, Risaralda,

Colombia.

aEstudiante de Medicina.

Estudio autofinanciado. Contribuciones de autoría: PMT tuvo la idea de investigación, diseñó el estudio, analizó e interpretó los datos, redactó el manuscrito y aprobó su publicación. RCK recolectó los datos, revisó y aprobó el manuscrito para su publicación. RPE interpretó los datos, redactó y aprobó el manuscrito para su publicación. AP recolectó los datos, revisó y aprobó el manuscrito para su publicación. AJRM diseño el estudio, interpretó los datos, revisó y aprobó el manuscrito para su publicación.

Conflictos de intereses: Los autores declararon no tenerlos en este manuscrito.

Recibido el 22 de enero de 2013, aceptado el 7 de marzo de 2013.

Correspondencia a: Percy Mayta-Tristán Av. Brasil \#2169, Dpto 802. Lima 11, Perú. Teléfono: (511) 987-532133 E-mail: percy.mayta@upc.edu.pe p.mayta@gmail.com

\section{Apreciación de estudiantes de Medicina latinoamericanos sobre la capacitación universitaria en investigación científica}

\author{
PERCY MAYTA-TRISTÁN ${ }^{1,2}$, RONNY CARTAGENA-KLEIN ${ }^{3}$, \\ RENEÉ PEREYRA-ELÍAS ${ }^{1,4, a}$, ALEJANDRA PORTILLO ${ }^{5}$, \\ ALFONSO J. RODRÍGUEZ-MORALES ${ }^{6,7}$
}

\section{Latin American medical students' appraisal on university scientific research training}

Background: Learning research skills should be a goal during undergraduate training of physicians. Aim: To identify Latin American medical students' appraisal on research skills university training. Material and Methods: A self-administered survey about experience in research, self-assessment of research skills, quality of training in the area received at the university and that importance of publishing as undergraduate students, was answered by 208 medical students aged $23 \pm 3$ years (54\% male), attending a medical students congress. Results: Seventy percent of respondents pertained to medical students' scientific societies and $34 \%$ had published in a scientific journal. Fifty two percent considered as good or very good the training level received at their universities on information retrieval and $45 \%$ considered good the training in research methodology. Thirty two percent considered as poor or none the training received in scientific writing and $37 \%$ in the publishing process. Eighty nine percent considered student publishing as important and $61 \%$ perceived limitations in this matter. Conclusions: The university training level received by Latin American medical students on research and publication process was evaluated as deficient by these students.

(Rev Med Chile 2013; 141: 716-722).

Key words: Latin America; Publications; Research; Students, medical; Universities.
L a investigación científica constituye uno de los cimientos en los cuales se sustenta la formación integral de un estudiante de medicina ${ }^{1}$. Sin embargo, es común que, en el contexto latinoamericano, la capacitación universitaria en asuntos científicos sea ensombrecida por la destinada a la actividad asistencial ${ }^{2}$. Con esto, se desarrolla y vigoriza una pobre cultura de investigación. Si se complementa este componente con la deficiente instrucción en redacción de artículos y su publicación ${ }^{1,3-5}$, se acondiciona el escenario perfecto que explica la baja producción científica de Latinoamérica en el área biomédica ${ }^{6}$.

Ante esta desfavorable situación, los estudiantes de medicina latinoamericanos se asociaron en organizaciones llamadas 'Sociedades Científicas de Estudiantes de Medicina', a través de las cuales se han desplegado diversas estrategias para menguar esta problemática ${ }^{7-9}$. Se deben mencionar las dos iniciativas principales: 1) La concepción y desarrollo de revistas científicas estudiantiles -medida que ha tomado fuerza y notoriedad progresivas en 
los últimos años, teniendo por objetivo principal la publicación de resultados originales, además de casos clínicos y cartas al editor ${ }^{10-13}$ y 2) la frecuente realización de programas de capacitación en búsqueda de información, lectura crítica, metodología de la investigación, bioestadística, redacción científica y publicación ${ }^{1}$.

En cuanto a las revistas científicas, es importante remarcar que estas brindan a los estudiantes un medio de calidad para la comunicación de los resultados de sus estudios, con lo cual se concluye el proceso de la investigación ${ }^{10}$. La Federación Latinoamericana de Sociedades Científicas de Estudiantes de Medicina (FELSOCEM) congrega a estas organizaciones en la región y vela por mantener la dirección de sus objetivos ${ }^{14}$. Esta institución tiene como medio de difusión científica oficial a la revista CIMEL (Ciencia e Investigación Médica Estudiantil), creada en 1995 y que tiene frecuencia semestral ${ }^{10}$. Además, existen revistas locales de las sociedades científicas de algunos países o universidades, como es el caso de la Asociación Nacional Científica de Estudiantes de Medicina de Chile (ANACEM), que publica una revista con el mismo nombre ${ }^{15}$.

Gracias a las estrategias mencionadas, se ha observado la considerable y creciente contribución de los estudiantes de medicina a la producción científica de varios países de la región ${ }^{16-19}$.

Los propios estudiantes perciben, en su mayoría, que la formación universitaria recibida en temas relacionados a la investigación (especialmente en redacción científica y publicación) es deficiente, según sugieren los resultados de estudios realizados en estudiantes de medicina de Perú ${ }^{20,21}$. No hemos encontrado estudios que evalúen el estado de la cuestión en estudiantes de medicina de otros países de Latinoamérica. El presente estudio tiene como objetivo identificar la apreciación de estudiantes de medicina latinoamericanos asistentes a un evento científico de la FELSOCEM, sobre el nivel de capacitación en esta área que reciben en la universidad y su nivel actual, así como determinar la importancia y las limitaciones percibidas para la publicación de artículos en el pregrado.

\section{Materiales y Métodos}

Se realizó un estudio de corte transversal en el mes de octubre de 2008, en el marco del XXIII
Congreso Científico Internacional de Estudiantes de Medicina (CCI) organizado por FELSOCEM, el cual se llevó a cabo en Iquique, Chile. El estudio fue aprobado por FELSOCEM.

La población que se incluyó en el estudio correspondió a estudiantes de medicina inscritos en dicho evento científico que participasen voluntariamente con el llenado de la encuesta, luego de la explicación de los objetivos del estudio. Se excluyó a aquellos sujetos de estudio que hayan devuelto las encuestas vacías.

Se utilizó una encuesta anónima y autoadministrada que incluía datos sociodemográficos y secciones que evaluaban las motivaciones para la publicación y la actividad científica y publicación. Este instrumento ha sido aplicado en investigación previa a estudiantes de medicina en Perú ${ }^{20}$. Comprende tres secciones: 1) Aspectos universitarios (procedencia, año de estudios) y experiencia en investigación (número de trabajos realizados, presentados a congresos científicos, listos para publicar y publicaciones en revistas científicas); 2) Apreciación sobre el nivel de capacitación en investigación y publicación recibido en su formación universitaria y el nivel actual de sus habilidades en este aspecto y 3) Aspectos relacionados con la importancia y limitaciones de la publicación en el pregrado.

El nivel de capacitación e investigación se evaluó a través de cinco ítems: búsqueda de información, lectura crítica, metodología de la investigación, redacción de artículos y proceso de publicación, en los que se preguntó sobre la capacitación que recibió durante la formación en pregrado a través de una escala de Likert de cinco alternativas: nula, deficiente, regular, buena y muy buena ${ }^{20}$ la cual tuvo una alfa de Crombach de 0,82 y para el caso de su nivel de capacitación actual usando los mismos ítems y alternativas se obtuvo un alfa de Crombach de 0,81.

Las encuestas se digitalizaron en una base de datos utilizando el programa Microsoft Excel ${ }^{\circledast}$. Previo control de calidad, se realizó el análisis de datos utilizando el paquete estadístico STATA 11.0. Se muestran las frecuencias absolutas y relativas. Se utilizó la prueba de Wilcoxon para evaluar las diferencias entre la percepción del nivel de capacitación recibido en pregrado y el nivel actual. La prueba U de Mann Withney fue utilizada para comparar el nivel de formación en pregrado y el nivel actual en investigación según 
procedencia, pertenencia a sociedad científica y antecedente de haber publicado.

La percepción de importancia y limitaciones de la publicación en pregrado, inicialmente evaluada mediante escala tipo Likert (totalmente en desacuerdo, en desacuerdo, de acuerdo, totalmente de acuerdo, no sabe/no opina), fueron dicotomizadas (de acuerdo + totalmente de acuerdo/ resto) y comparadas con las variables procedencia, antecedente de publicación o pertenencia a sociedad científica utilizando la prueba de $\chi^{2}$ (chi cuadrado). Se consideró el valor $\mathrm{p}<0,05$ como estadísticamente significativo.

\section{Resultados}

La encuesta fue alcanzada a 211 estudiantes de primero a séptimo año de la carrera de medicina; se excluyeron tres por devolver las encuestas vacías, quedando 208 encuestas válidas para el análisis de datos.

\section{Características de la población}

Se incluyó sujetos de estudio de ocho países de América Latina (Tabla 1) y 35 universidades.

La mayoría $(70,3 \%)$ pertenecía a una sociedad científica de estudiantes de medicina. La tercera parte refirió haber publicado en alguna revista científica (Tabla 1), y la mayoría (57\%) conoce al menos a un compañero que ha publicado un trabajo de investigación.

Treinta y ocho coma ocho por ciento conocía al menos una revista estudiantil. CIMEL era conocida por $13,5 \%$ del total de encuestados y la Revista ANACEM era conocida por $20,4 \%$ de estudiantes chilenos.

\section{Capacitación y habilidades en investigación}

Cincuenta y dos por ciento de los encuestados estimaron como bueno o muy bueno el nivel de capacitación recibido en sus universidades en cuanto a búsqueda de información, $45 \%$ en metodología de la investigación y $36 \%$ en redacción de artículos y proceso de publicación. Asimismo, $32 \%$ consideró nulo o deficiente el entrenamiento recibido en redacción de artículos y $37 \%$ en cuanto al proceso de publicación (Figura 1A).

Sesenta y cinco por ciento de los estudiantes consideró bueno o muy bueno su actual nivel en búsqueda de información, $46 \%$ en redacción de artículos y 40\% en el proceso de publicación. Diecinueve por ciento valoró nula o deficiente su capacidad de desarrollar el proceso de publicación (Figura 1B).

Los alumnos percibieron que su nivel actual era superior al nivel de la capacitación recibida en la universidad $(\mathrm{p}<0,001)$ para los tópicos de búsqueda de información, lectura crítica, redacción de artículos y el proceso de publicación. En el caso de metodología de la investigación, consideraron que tienen el mismo nivel que el recibido por su universidad (Wilcoxon, $\mathrm{p}>0,05$ ).

Según el país de procedencia (tomando Chile

Tabla 1. Características de los sujetos de estudio

\begin{tabular}{|c|c|c|}
\hline Característica & $\mathbf{n}$ & (\%) \\
\hline Varones & 113 & $(54,3)$ \\
\hline Edad (años, media \pm desviación estándar) & 22,9 & $\pm 2,9$ \\
\hline \multicolumn{3}{|l|}{ País (n = 207) } \\
\hline Chile & 101 & $(48,8)$ \\
\hline Bolivia & 38 & $(18,4)$ \\
\hline Venezuela & 36 & $(17,4)$ \\
\hline Perú & 15 & $(7,3)$ \\
\hline Paraguay & 8 & $(3,9)$ \\
\hline Panamá & 5 & $(2,4)$ \\
\hline México & 3 & $(1,5)$ \\
\hline Honduras & 1 & $(0,5)$ \\
\hline \multicolumn{3}{|l|}{ Año de estudio $(n=185)$} \\
\hline Primer año & 15 & $(8,1)$ \\
\hline Segundo año & 27 & $(14,6)$ \\
\hline Tercer año & 40 & $(21,6)$ \\
\hline Cuarto año & 36 & $(19,5)$ \\
\hline Quinto año & 33 & $(17,8)$ \\
\hline Sexto año & 23 & $(12,4)$ \\
\hline Séptimo año & 11 & $(6,0)$ \\
\hline \multicolumn{3}{|l|}{ Actividad científica $(n=208)$} \\
\hline Pertenece a sociedad científica & 137 & $(70,3)$ \\
\hline Tiene al menos un trabajo no publicado & 142 & $(68,3)$ \\
\hline Ha presentado resúmenes en congresos & 110 & $(52,9)$ \\
\hline Tiene actualmente un trabajo para publicar & 84 & $(42,2)$ \\
\hline Ha publicado en una revista científica & 70 & $(33,7)$ \\
\hline Ha publicado en revistas nacionales & 65 & $(31,3)$ \\
\hline Ha publicado en revistas internacionales & 31 & $(14,9)$ \\
\hline \multicolumn{3}{|l|}{ Inicio en publicación ( $n=70$ ) } \\
\hline Junto a un docente investigador & 26 & $(37,1)$ \\
\hline Con su grupo de investigación & 24 & $(34,3)$ \\
\hline Solo & 20 & $(28,6)$ \\
\hline
\end{tabular}



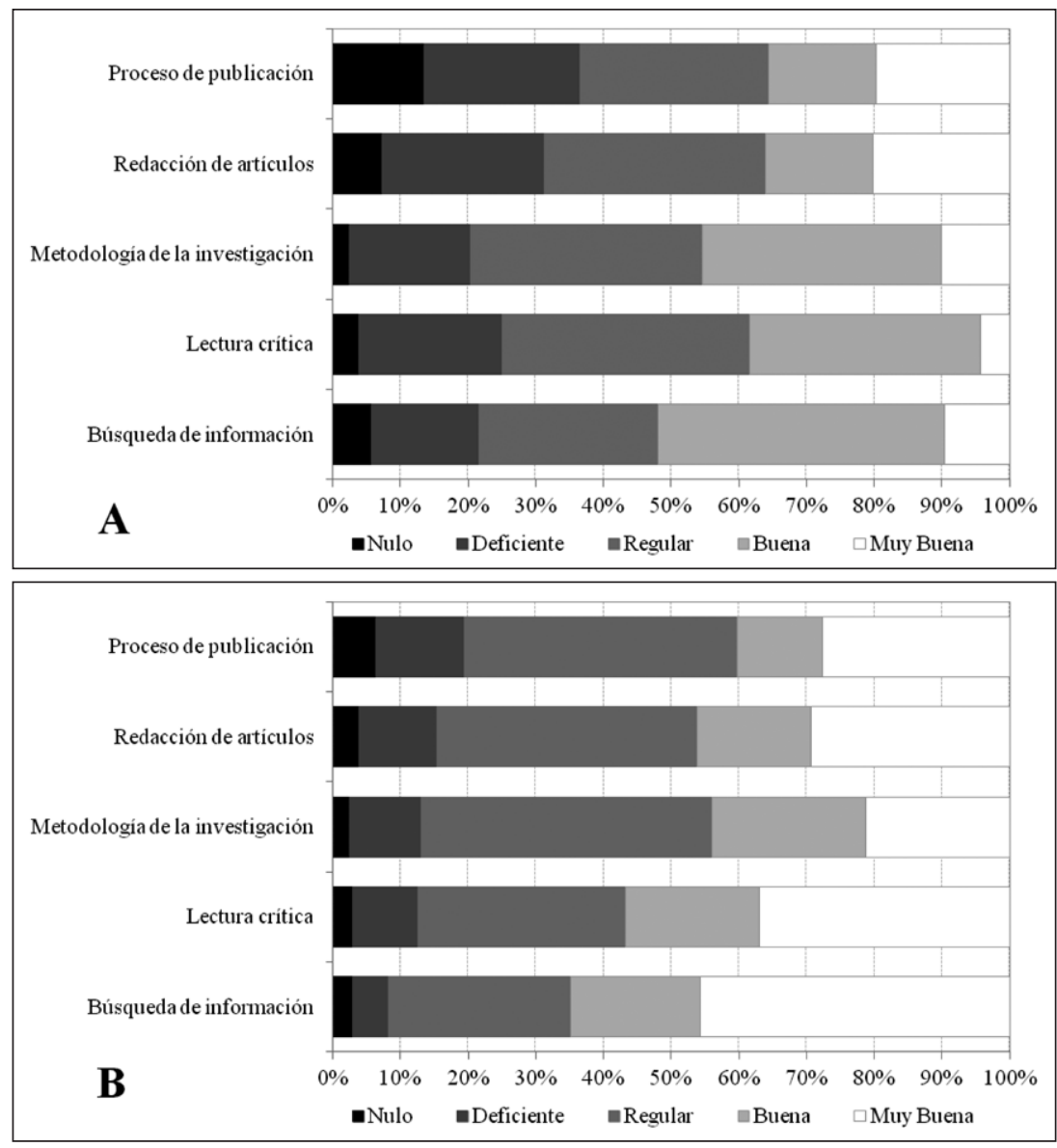

Figura 1. Percepción sobre la capacitación que ha recibido sobre investigación durante su formación en pregrado (A) y nivel actual para desarrollarse (B).

como referencia), no se encontraron diferencias en la percepción del nivel de la capacitación recibida por la universidad ( $p>0,05)$. Sin embargo, en la percepción del nivel de las habilidades propias para la investigación, los estudiantes chilenos señalaron tener un mayor nivel en búsqueda de información $(\mathrm{p}=0,004)$, lectura crítica $(\mathrm{p}=0,001)$ $y$ el proceso de publicación $(\mathrm{p}=0,020)$.

Los estudiantes que pertenecían a una sociedad científica de estudiantes de medicina percibieron mayor nivel de capacitación recibida en la universidad en cuanto a búsqueda de información $(\mathrm{p}=0,002)$. No obstante, en cuanto a la percepción de sus habilidades, refirieron presentar un nivel superior para la búsqueda de información $(\mathrm{p}=0,003)$, lectura crítica $(\mathrm{p}=0,001)$, metodología de la investigación $(\mathrm{p}=0,014)$ y proceso de publicación $(\mathrm{p}=0,020)$ comparados con los que no pertenecían a sociedades científicas.
Según el antecedente de haber publicado, se encontraron diferencias en la percepción de lo brindado por la universidad. Quienes lo habían hecho refirieron que sus universidades les brindaron mayores conocimientos en búsqueda de información $(\mathrm{p}=0,002)$, metodología de la investigación $(\mathrm{p}=0,034)$, redacción de artículos $(\mathrm{p}=0,009)$ y en el proceso de publicación $(\mathrm{p}=0,049)$. Quienes habían publicado también presentaron una apreciación de un nivel superior en todos los procesos de investigación consultados, respecto de los que no habían publicado $(\mathrm{p}<0,001)$.

Ochenta y ocho coma siete por ciento consideró que la publicación en el pregrado es importante; mejorar el nivel de lectura crítica en investigación fue el motivo señalado con mayor frecuencia (Tabla 2). No se encontraron diferencias para la percepción sobre la importancia de la publicación 
Tabla 2. Percepciones de estudiantes de medicina sobre la producción científica en pregrado

\begin{tabular}{|c|c|c|}
\hline \multirow[t]{2}{*}{ Percepciones } & \multicolumn{2}{|c|}{ De acuerdo } \\
\hline & n* & (\%) \\
\hline \multicolumn{3}{|l|}{ ¿Por qué consideran que es importante publicar desde el pregrado? } \\
\hline ... mejoro mi lectura crítica de investigación & 144 & 93,5 \\
\hline ... contribuyo a la producción científica nacional & 122 & 79,7 \\
\hline ... es la única forma de que me reconozcan como investigador & 107 & 70,4 \\
\hline ... formo un hábito personal y contribuyo a una cultura de publicación en mi entorno & 107 & 69,0 \\
\hline ... hago posible que otros investigadores conozcan mis resultados y puedan retroalimentarme & 103 & 66,0 \\
\hline ... refleja la valoración que tengo por la investigación que he realizado & 79 & 51,3 \\
\hline ... así culmino con el proceso de investigación & 55 & 36,2 \\
\hline \multicolumn{3}{|l|}{ ¿Qué limitaciones tienen para publicar sus investigaciones? } \\
\hline ... por falta de apoyo docente & 25 & 25,8 \\
\hline ... no tengo incentivos a nivel académico & 20 & 23,8 \\
\hline ... por falta de tiempo & 19 & 18,8 \\
\hline ... tengo problemas para el análisis de datos & 15 & 18,1 \\
\hline ... no he sido capacitado en redacción científica & 17 & 17,3 \\
\hline ... desconozco el proceso de envío y seguimiento de mis artículos a una revista & 15 & 16,0 \\
\hline ... no manejo el inglés como para publicar en revistas extranjeras & 11 & 15,3 \\
\hline ... no tengo una cultura de publicación & 13 & 14,3 \\
\hline ... desconozco en que revistas puedo publicar & 13 & 14,0 \\
\hline ... la calidad de mis estudios no es para que sean publicados & 7 & 10,1 \\
\hline
\end{tabular}

* El número de sujetos que respondieron cada alternativa no fue el mismo.

según país de procedencia o año de estudios, pero sí en relación al antecedente de haber publicado $\left(\chi^{2}, \mathrm{p}=0,017\right)$ o pertenecer a una sociedad científica $\left(\chi^{2}, \mathrm{p}=0,023\right)$.

Para 60,8\% de los encuestados, existen limitaciones para publicar desde el pregrado, siendo la falta de apoyo docente e incentivos académicos los indicados como principales. Noventa por ciento no consideró la calidad de su estudio como un factor limitante (Tabla 2). No se encontraron diferencias según país de procedencia, año de estudio o antecedente de publicación en la percepción de las limitaciones. Sin embargo, aquellos que pertenecen a una sociedad científica manifestaron, en mayor proporción, que existen limitaciones para $\operatorname{publicar}\left(\chi^{2}, \mathrm{p}=0,013\right)$.

\section{Discusión}

La población del estudio estuvo conformada por estudiantes de medicina interesados en el área científica. Esto se deduce porque son asistentes a un congreso científico de la FELSOCEM (que tiene como eje central un concurso de trabajos de investigación), además de la alta frecuencia de presentación de trabajos en congresos científicos previos (53\%), pertenencia a su sociedad científica local (70\%) y antecedente de publicación (34\%). Por otro lado, la percepción de manejar un mayor nivel que el recibido en su formación universitaria en diversas habilidades en investigación podría indicar un entrenamiento extracurricular muy probablemente relacionado a las sociedades científicas. Estas características limitan la posible representatividad de la población estudiada para los estudiantes de medicina en general, porque sólo se evaluaron estudiantes motivados en investigación.

Similar a lo reportado previamente $e^{20,21}$, nuestro estudio encontró que los estudiantes de medicina consideraron deficiente la preparación universitaria recibida en temas relacionados a investigación, especialmente en lo referido a la redacción de artículos y su publicación, lo cual no fue tan notorio en cuanto a búsqueda de información, metodología de la investigación. Esto se debe a que el entrenamiento universitario en el proceso de investigación se limita a enseñar primordial- 
mente aspectos metodológicos y usualmente no se incluye la cristalización de este proceso mediante la publicación $\mathrm{n}^{3,22}$.

Por otro lado, la frecuencia y magnitud de dicha insatisfacción en los sujetos estudiados es considerablemente menor a los antecedentes en Perú ${ }^{20,21}$. Esto se puede explicar por la presencia de estudiantes de otras nacionalidades que hayan recibido una formación universitaria más orientada a aspectos científicos, lo cual se evidencia en la baja producción científica de Perú comparada con otros países de la región ${ }^{6}$.

El hallazgo de un mayor nivel percibido por los sujetos de estudio respecto del recibido en su formación universitaria para la mayoría de los tópicos es un posible reflejo del producto de las medidas implementadas por las sociedades científicas ${ }^{9}$. El que no exista diferencias exclusivamente en cuanto a metodología de la investigación, se puede deber a la mayor orientación que las universidades brindan a este aspecto ${ }^{3,22}$. Además, se encontró que los estudiantes que pertenecían a sociedades científicas percibían un nivel superior en sus habilidades de investigación (excepto en búsqueda de información), respecto de los que no pertenecían. Estos resultados permiten apreciar el impacto beneficioso de estas instituciones en llenar la brecha deficitaria que supone la educación en investigación recibida en el pregrado ${ }^{7-9,23}$.

Asimismo, los estudiantes chilenos refirieron presentar un mayor nivel de búsqueda de información, lectura crítica y proceso de publicación respecto de los estudiantes de otras nacionalidades. La producción científica de Chile en general y el aporte del pregrado son significativamente mayores que en otros países de la región (PoggiSanz $\mathrm{P}$ et al, observaciones no publicadas), lo cual concuerda con estos resultados.

Las limitaciones percibidas son mayores en los estudiantes asociados a una sociedad científica, lo cual se podría explicar por la posibilidad de encontrar más frecuentemente barreras y limitaciones relacionadas con la mayor actividad científica realizada por estos alumnos.

Nuestro estudio tiene limitaciones: como se explicó previamente, se tomó una muestra por conveniencia de estudiantes de medicina motivados en el área científica, por lo cual los resultados no son extrapolables para la población general de estudiantes de medicina de América Latina.

Este estudio da una aproximación, desde el punto de vista del estudiante interesado, sobre la formación en investigación en el pregrado médico en Latinoamérica. Futuros estudios deberían evaluar en forma integral e individualizada cada programa de formación en investigación, pudiendo tomar el modelo de evaluación de las acciones formativas planteado por Kirkpatrick ${ }^{24,25}$ que incluye la reacción, aprendizaje, comportamientos y resultados. Que para el caso de investigación, puede ir desde las percepciones de los estudiantes (que evaluamos en este estudio), hasta los cambios curriculares que se pueden implementar y el resultado final que sería el número de publicaciones realizadas por los alumnos.

Concluimos que la formación universitaria en temas relacionados con la investigación es percibida como deficiente por los estudiantes de pregrado de Latinoamérica estudiados y que el nivel propio referido es significativamente más alto. Al ser esto último una probable consecuencia de las estrategias desplegadas por las sociedades científicas, recomendamos que se brinde un mayor apoyo a estas organizaciones. De esta forma se podrá mejorar no sólo la apreciación, como la observada en este estudio, sino incrementar y fomentar más las actividades de investigación que puedan conducir a la publicación científica, que debe motivarse desde pregrado, como ha sido discutido ampliamente en los últimos años ${ }^{1,5,26}$.

\section{Referencias}

1. Gutiérrez C, Mayta P. Publicación desde el Pre Grado en Latinoamérica: importancia, limitaciones y alternativas de solución. CIMEL 2003; 8: 54-60.

2. Rojas-Revoredo V. Las publicaciones en revistas indexadas, único indicador de la producción de las sociedades científicas estudiantiles. CIMEL 2007; 12: 5-6.

3. Laufer M. Enseñar cómo publicar. Interciencia 2008; 33: 321.

4. Salinas JL, Vildózola H. Investigación en pregrado: dificultades y posibles soluciones. An Fac Med 2008; 69: 216.

5. Huamaní C, Mayta-Tristán P, Rodríguez-Morales AJ. Publicar desde pregrado. Interciencia 2008; 33: 785.

6. Huamaní C, Gonzáles G, Curioso WH, Pacheco-Romero J. Redes de colaboración y producción científica Sudamericanas en medicina clínica, ISI Current Contents 200-2009. Rev Med Chile 2012; 140: 466-75.

7. Mayta-Tristán P. Sociedades científicas de estudiantes de 
medicina: el futuro de la investigación en Latinoamérica. Med UNAB 2010; 13: 3-4.

8. Arce-Villavicencio Y, Astuvilca-Cupe J. Grupos Estudiantiles de Investigación: una prioridad en las sociedades científicas estudiantiles de Latinoamérica. CIMEL. 2007; 12: $45-6$

9. Pereyra-Elías R, Rodríguez-Morales A, Mayta-Tristán P. Undergraduate publication in Latin America: Role of medical student's scientific societies. Med Teach 2011; 33: 594.

10. Mayta P. Revistas científicas estudiantiles en Latinoamérica. Rev Med Chile 2006; 134: 395-7.

11. Ángulo-Bazán Y. Situación actual de las revistas científicas estudiantiles en Latinoamérica. CIMEL 2009; 13: 36-7.

12. Orostegui-Pinilla D, Cabrera-Samith I, Ángulo-Bazán Y, Mayta-Tristán P, Rodríguez-Morales AJ. Encuentros internacionales de revistas científicas estudiantiles de las ciencias de la salud en Latinoamérica, 1998-2008. Rev Panam Salud Pública 2009; 25: 469-70.

13. Cabrera-Samith I, Oróstegui-Pinilla D, Angulo-Bazán Y, Mayta-Tristán P, Rodríguez-Morales AJ. Revistas científicas de estudiantes de medicina en Latinoamérica. Rev Med Chile 2010; 138: 1451-5.

14. Niño R, Marañón R, Rodríguez-Morales AJ. FELSOCEM: Visión científica de un pasado, un presente y un futuro. CIMEL 2003; 8: 61-2.

15. Cabrera-Samith I, Garrido F. El desarrollo de las revistas científicas de estudiantes de medicina en Chile. Rev Med Chile 2009; 137: 1265-6.

16. Huamaní C, Chávez-Solís P, Mayta-Tristán P. Aporte estudiantil en la publicación de artículos científicos en revistas médicas indizadas en SciELO-Perú, 1997-2005. An Fac Med (Lima) 2008; 69: 42-5.

17. Taype-Rondán A, Lajo-Arauzo Y, Gutiérrez-Brown R,
Zamalloa-Masias N, Saldaña-Gonzales M. Aporte de las sociedades estudiantiles en la publicación científica en SciELO-Perú, 2009-2010. Rev Perú Med Exp Salud Pública 2011; 28: 691-2.

18. Angulo R, Angulo F, Huamaní C, Mayta-Tristán P. Publicación estudiantil en revistas médicas venezolanas 2001-2005. CIMEL 2008; 13: 6-8.

19. Pachajoa-Londoño HM. Publicación de artículos originales desde el pregrado en una revista médica colombiana entre 1994-2004. CIMEL 2006; 11:24-6.

20. Molina-Ordóñez J, Huamaní C, Mayta-Tristán P. Apreciación estudiantil sobre la capacitación universitaria en investigación: Estudio preliminar. Rev Perú Med Exp Salud Pública 2008; 25: 325-9.

21. Mayta-Tristán P, Peña-Osuvilca A. Importancia de la publicación en las sociedades científicas de estudiantes de medicina del Perú: estudio preliminar. CIMEL 2009; 14: 27-34.

22. Pamo O. Publicar o perecer. Boletín de la Sociedad Peruana de Medicina Interna 2000; 13: 1.

23. Huamani C, Chávez-Solís P, Domínguez-Haro W, Solano-Aldana M. Producción científica estudiantil: Análisis y expectativas. Rev Perú Med Exp Salud Pública 2007; 24: 444-6.

24. Triviño X, Sirhan M, Moore P, Montero L. Impacto de un programa de formación en docencia en una escuela de medicina. Rev Med Chile 2011; 139: 1508-15.

25. Steinert Y, Mann K, Centeno A, Dolmans D, Spencer J, Gelula M. A systematic review of faculty development initiatives designed to improve teaching effectiveness in medical education: BEME Guide No. 8. Med Teach 2006; 28: 497-526

26. Escudero-Sepúlveda A, Escudero-Sepúlveda J, Rodríguez-Morales AJ. La redacción de un trabajo científico. Invest Clin 2012; 53: 111-2. 